PROCEEDINGS OF THE

AMERICAN MATHEMATICAL SOCIETY

Volume 126, Number 6, June 1998, Pages 1621-1626

S 0002-9939(98)04205-1

\title{
ON SOME FINITELY BASED REPRESENTATIONS OF SEMIGROUPS
}

\author{
NIKOLAY N. SILKIN
}

(Communicated by Lance W. Small)

\begin{abstract}
In this paper we present a method of obtaining finitely based linear representations of possibly infinitely based semigroups.
\end{abstract}

Let $R\left\{x_{1}, x_{2}, \ldots\right\}$ be a free associative algebra over a commutative ring $R$ with the countable set of free generators $\left\{x_{1}, x_{2}, \ldots\right\}$. An endomorphism $\alpha$ of $R\left\{x_{1}, x_{2}, \ldots\right\}$ is called a semigroup endomorphism if $x_{1} \alpha, x_{2} \alpha, \ldots$ are monomials (i.e. finite products of $x_{i}$ 's). An ideal $I$ of $R\left\{x_{1}, x_{2}, \ldots\right\}$ is called an $S$-ideal if $I$ is closed under all semigroup endomorphisms of $R\left\{x_{1}, x_{2}, \ldots\right\}$. Let $S$ be a semigroup, $M$ a faithful module over $R$. A multiplicative homomorhism $\psi: S \longrightarrow \operatorname{End}_{R} M$ is called a (linear) representation of $S$ on $M$. An element $p=p\left(x_{1}, x_{2}, \ldots, x_{n}\right)$ of $R\left\{x_{1}, x_{2}, \ldots\right\}$ is called an identity of the representation $\psi$ if

$$
p\left(\psi\left(s_{1}\right), \ldots, \psi\left(s_{n}\right)\right)=0 \text { for all } s_{1}, \ldots, s_{n} \in S .
$$

It is not hard to show that the set of all identities of any representation is an $S$ ideal of $R\left\{x_{1}, x_{2}, \ldots\right\}$. An $S$-ideal $I$ of $R\left\{x_{1}, x_{2}, \ldots\right\}$ is called finitely $S$-generated if there exist $p_{1}, \ldots, p_{k} \in I$ such that $I$ is the least $S$-ideal of $R\left\{x_{1}, x_{2}, \ldots\right\}$ containing $p_{1}, \ldots, p_{k}$. A representation $\psi$ is called finitely based if the $S$-ideal $I$ of its identities is finitely $S$-generated. Any set $\left\{p_{1}, \ldots, p_{k}\right\} \subseteq I$ such that the elements $p_{1}, \ldots, p_{k}$ $S$-generate $I$ is called a finite basis of identities of $\psi$.

S.M.Vovsi and N.H.Shon in [VSh] proved that every representation of a finite group over a field is finitely based. A semigroup version of this problem is open: it is not known whether every representation of a finite semigroup is finitely based.

Let $A$ be an associative algebra over $R, S=\langle A, \cdot\rangle$ its multiplicative semigroup, and $M$ be an $R$-module. Let $\phi: A \longrightarrow \operatorname{End}_{R} M$ be a homomorphism ( $R$-linear, additive, and multiplicative). The kernel of $\phi, \operatorname{Ker} \phi$, is a two-sided ideal in $A$. Define a representation $\psi$ of $S$ on $M$ by $m \psi(s)=m \phi(s)$ for all $m \in M, s \in S$. The representation $\psi$ is a linear representation of $S$ on $M$. Call the representation $\psi$ associated with $\phi$.

From now on we assume that the ring $R$ and the module $M$ have at least one of the following properties: (i) for any integer $m$ there exist $r_{1}, \ldots, r_{m} \in R$ such that $\prod_{i<j}\left(r_{i}-r_{j}\right) m=0$ implies $m=0$ for each $m \in M$; (ii) the ring $R$ is finitely generated. In this paper we prove the following theorem.

Received by the editors June 17, 1996 and, in revised form, November 19, 1996.

1991 Mathematics Subject Classification. Primary 20M89, 16R50.

Key words and phrases. Semigroups, identities, linear representations.

(c) 1998 American Mathematical Society 
Theorem 1. Let $A$ be an associative ring, $S=\langle A, \cdot\rangle$ its multiplicative semigroup, $M$ an $R$-module, and $R$ and $M$ satisfy at least one of the conditions (i) and (ii) mentioned above. Suppose $\phi: A \longrightarrow \operatorname{End}_{R} M$ is a homomorphism (R-linear, additive, and multiplicative). Then the associated representation $\psi$ of $S$ on $M$ is finitely based if and only if there is a finite basis of identities of the ring $A / \operatorname{Ker} \phi$.

L.Al'shanskii and A.Kushkuley proved in $[\mathrm{AK}]$ that the natural representation of the (infinitely based as a semigroup) 6-element Brandt monoid is finitely based. Theorem 1 above provides a number of similar examples. So, one can use finite basis results from ring theory to get the finite basis property for some semigroup representations. For instance, it follows from Theorem 1 that the regular representation (by right multiplications) of the multiplicative semigroup $S$ of all square $n \times n$ matrices, $n>1$, over a finite associative ring with 1 is finitely based, while it is known that this semigroup has no finite basis of its identities, see [S]. Note that the associative algebra of all square $n \times n$ matrices, $n>1$, over a finite associative ring is finitely based, see $[\mathrm{L}, \mathrm{K}]$.

Recall that a polynomial $p=p\left(x_{1}, \ldots, x_{n}\right) \in R\left\{x_{1}, x_{2}, \ldots\right\}$ is called an identity of an associative algebra $A$ over a ring $R$ if $p\left(a_{1}, a_{2}, \ldots, a_{n}\right)=0$ for all $a_{1}, \ldots, a_{n} \in$ $A$. The set of all identities of the algebra $A$ forms a $T$-ideal of $R\left\{x_{1}, x_{2}, \ldots\right\}$ (i.e. an ideal which is closed under all endomorphisms of $\left.R\left\{x_{1}, x_{2}, \ldots\right\}\right)$. Note that each $T$-ideal is also an $S$-ideal. Recall also that the first linearization in $x_{i}$ of a polynomial $p=p\left(x_{1}, \ldots, x_{n}\right) \in R\left\{x_{1}, x_{2}, \ldots\right\}$ is the polynomial

$$
\begin{aligned}
& q\left(x_{1}, \ldots, x_{n}, x_{n+1}\right) \\
& =p\left(x_{1}, \ldots, x_{i-1}, x_{i}+x_{n+1}, x_{i+1}, \ldots, x_{n}\right) \\
& -p\left(x_{1}, \ldots, x_{i-1}, x_{i}, x_{i+1}, \ldots, x_{n}\right) \\
& -p\left(x_{1}, \ldots, x_{i-1}, x_{n+1}, x_{i+1}, \ldots, x_{n}\right) .
\end{aligned}
$$

Call a polynomial $q$ a linearization of a polynomial $p$ if there is a finite sequence of polynomials $p=p_{1}, p_{2}, \ldots, p_{k}=q$ such that for each $i=2, \ldots, k$ the polynomial $p_{i}$ is the first linearization of $p_{i-1}$ in some indeterminate $x_{l}$. It follows from the definition of a $T$-ideal that any $T$-ideal is closed under taking linearizations. Note that the set of all possible linearizations of a polynomial is finite up to renumberings of the indeterminates. (For a thorough account of linearizations see, for example, [R1].)

Let $r_{1}, \ldots, r_{s} \in R$. Bring in a set of transformations related to $r_{1}, \ldots, r_{s}$. Let $p=p\left(x_{1}, \ldots, x_{n}\right)$ be a polynomial and let $d_{i}$ be the degree of $p$ in $x_{i}$. Write

$$
p=p_{0}+p_{1}+\cdots+p_{d_{i}},
$$

where the polynomials $p_{0}, \ldots, p_{d_{i}}$ are homogeneous in $x_{i}$ with $\operatorname{deg}\left(p_{j}\right)=j$ for all j. So,

$$
\begin{aligned}
& p\left(x_{1}, \ldots, x_{i-1}, r_{j} x_{i}, x_{i+1}, \ldots, x_{n}\right) \\
& =p_{0}\left(x_{1}, \ldots, x_{i-1}, x_{i}, x_{i+1}, \ldots, x_{n}\right) \\
& +r_{j} p_{1}\left(x_{1}, \ldots, x_{i-1}, x_{i}, x_{i+1}, \ldots, x_{n}\right) \\
& +\cdots+r_{j}^{d_{i}} p_{d_{i}}\left(x_{1}, \ldots, x_{i-1}, x_{i}, x_{i+1}, \ldots, x_{n}\right) .
\end{aligned}
$$

Then the polynomial

$$
\begin{aligned}
& q\left(x_{1}, \ldots, x_{i-1}, x_{i}, x_{i+1}, \ldots, x_{n}\right) \\
& =r_{j}^{d_{i}} p\left(x_{1}, \ldots, x_{i-1}, x_{i}, x_{i+1}, \ldots, x_{n}\right) \\
& -p\left(x_{1}, \ldots, x_{i-1}, r_{j} x_{i}, x_{i+1}, \ldots, x_{n}\right)
\end{aligned}
$$


is called the homogenization of $p$ in $x_{i}$ relative to $r_{j}$. Call a polynomial $q$ a homogenization of $p$ if $q$ is a result of several consecutive homogenizations of $p$. It follows from the definition of a $T$-ideal that any $T$-ideal is closed under taking homogenizations. Note that the set of all possible homogenizations of a polynomial is finite up to renumberings of the indeterminates (since the set $r_{1}, \ldots, r_{s}$ is finite).

Lemma 1. A T-ideal is finitely generated (as a T-ideal) if and only if it is finitely S-generated.

Proof. Suppose that a $T$-ideal $I$ is finitely generated as a $T$-ideal by the finite set of polynomials $P=\left\{p_{1}, \ldots, p_{n}\right\}$. This means that the set of polynomials of the form $p\left(f_{1}, \ldots, f_{i}\right)$, where $p \in P, i$ is an integer, and $f_{1}, \ldots, f_{i}$ are arbitrary polynomials, generates $I$ as a two-sided ideal. Our goal is to find a finite set of polynomials $P^{\prime}=\left\{p_{1}^{\prime}, \ldots, p_{m}^{\prime}\right\}$ which $S$-generates $I$. This means in turn that the set of polynomials of the form $p^{\prime}\left(m_{1}, \ldots, m_{j}\right)$, where $p^{\prime} \in P^{\prime}, j$ is an integer, and $m_{1}, \ldots, m_{j}$ are arbitrary monomials (but not arbitrary polynomials!), generates $I$ as a two-sided ideal.

Since $I$ is a $T$-ideal, $I$ is closed under renaming indeterminates. So, without loss of generality we may assume that the polynomials $p_{1}, \ldots, p_{n}$ involve only indeterminates $x_{1}, \ldots, x_{k}$. Let $P_{1}$ be the set of all linearizations of polynomials $\left\{p_{1}, \ldots, p_{n}\right\}$ together with the polynomials $p_{1}, \ldots, p_{n}$ themselves. Since a polynomial has only finitely many linearizations, the set $P_{1}$ is finite. The definition of the linearization (1) can be rewritten as

$$
\begin{aligned}
& p\left(x_{1}, \ldots, x_{i-1}, x_{i}+x_{n+1}, x_{i+1}, \ldots, x_{n}\right) \\
& =q\left(x_{1}, \ldots, x_{n}, x_{n+1}\right) \\
& -p\left(x_{1}, \ldots, x_{i-1}, x_{i}, x_{i+1}, \ldots, x_{n}\right) \\
& -p\left(x_{1}, \ldots, x_{i-1}, x_{n+1}, x_{i+1}, \ldots, x_{n}\right) .
\end{aligned}
$$

Let $p=p_{i}\left(x_{1}, \ldots, x_{k}\right) \in\left\{p_{1}, \ldots, p_{n}\right\}$. In particular, the formula (4) implies that

$$
\begin{aligned}
& p_{i}\left(\alpha_{1} m_{1}, \ldots, \alpha_{i-1} m_{i-1}, \beta_{1} w_{1}+\beta_{2} w_{2}, \alpha_{i+1} m_{i+1}, \ldots, \alpha_{k} m_{k}\right) \\
& =q\left(\alpha_{1} m_{1}, \ldots, \alpha_{i-1} m_{i-1}, \beta_{1} w_{1}, \alpha_{i+1} m_{i+1}, \ldots, \alpha_{k} m_{k}, \beta_{2} m_{2}\right) \\
& -p_{i}\left(\alpha_{1} m_{1}, \ldots, \alpha_{i-1} m_{i-1}, \beta_{1} w_{1}, \alpha_{i+1} m_{i+1}, \ldots, \alpha_{k} m_{k}\right) \\
& -p_{i}\left(\alpha_{1} m_{1}, \ldots, \alpha_{i-1} m_{i-1}, \beta_{2} w_{2}, \alpha_{i+1} m_{i+1}, \ldots, \alpha_{k} m_{k}\right)
\end{aligned}
$$

for any choice of $\alpha_{1}, \ldots, \alpha_{k}, \beta_{1}, \beta_{2} \in R$ and arbitrary monomials $m_{1}, \ldots, m_{k}$, $w_{1}, w_{2}$. Now let $f_{1}, \ldots, f_{k}$ be arbitrary polynomials. Look at $p_{i}\left(f_{1}, \ldots, f_{k}\right)$. By induction on the total number of ' + ' signs in polynomials $f_{1}, \ldots, f_{k}$ one shows that the polynomial $p_{i}\left(f_{1}, \ldots, f_{k}\right)$ can be written as a linear combination of polynomials of the form $q\left(\alpha_{1} m_{1}, \ldots, \alpha_{l} m_{l}\right)$, where the polynomial $q$ is a linearization of $p_{i}, l$ is an integer, $\alpha_{1}, \ldots, \alpha_{l} \in R, m_{1}, \ldots, m_{l}$ are monomials and, moreover, for each $i=1, \ldots, l$ the term $\alpha_{i} m_{i}$ is an additive term of some $f_{j}, j=1, \ldots, k$. The base for this induction is provided by formula (5). The induction step is also implicit in formula (4). (Suppose $f_{i}=g_{1}+g_{2}$ is a nontrivial sum of two polynomials. Then in (4) one needs to set $x_{1}=f_{1}, \ldots, x_{i-1}=f_{i-1}, x_{i}=g_{1}, x_{i+1}=f_{i+1}$, $\ldots, x_{k}=f_{k}, x_{k+1}=g_{2}$.) So, for arbitrary polynomials $f_{1}, \ldots, f_{k}$ the polynomial $p_{i}\left(f_{1}, \ldots, f_{k}\right)$ can be written as a linear combination of polynomials of the form $q\left(\alpha_{1} m_{1}, \ldots, \alpha_{l} m_{l}\right)$, where $q \in P_{1}, \alpha_{1}, \ldots, \alpha_{l} \in R$, and $m_{1}, \ldots, m_{l}$ are monomials. Therefore, the $T$-ideal $I$ is generated as a two-sided ideal by the set of all polynomials of the form $p\left(\alpha_{1} m_{1}, \ldots, \alpha_{l} m_{l}\right)$, where $p \in P_{1}, \alpha_{1}, \ldots, \alpha_{l} \in R, l$ is an integer, and $m_{1}, \ldots, m_{l}$ are monomials. 
Case 1. The ring $R$ and the module $M$ satisfy condition (i). In this case, the Vandermonde argument (see for example [R1, R2]) can be applied to polynomials from $P_{1}$ to show that the $T$-ideal $I$ can be generated by a finite number of completely homogeneous polynomials. (A polynomial is completely homogeneous if all its monomials have the same degree in each indeterminate.) In fact, the Vandermonde argument shows that in this case if a polynomial belongs to the $T$-ideal $I$, then all completely homogeneous components of this polynomial (i.e. the sums of all monomials (together with their coefficients) having the same degree in each indeterminate) also belong to the $T$-ideal $I$. So in this case, let $P_{2}$ be the set of all completely homogeneous components of polynomials from $P_{1}$. Note that the set $P_{2}$ is finite. Moreover, if $p=p\left(x_{1}, \ldots, p_{k}\right) \in P_{2}$, and $\operatorname{deg}_{i} p_{1}=d_{i}$, then for arbitrary $\alpha_{1}, \ldots, \alpha_{k} \in R$ we have $p\left(\alpha_{1} x_{1}, \ldots, \alpha_{k} x_{k}\right)=\alpha_{1}^{d_{1}} \cdots \alpha_{k}^{d_{k}} p\left(x_{1}, \ldots, x_{k}\right)$, since $p$ is completely homogeneous. Thus the $T$-ideal $I$ can be generated as a two-sided ideal by the set of all polynomials of the form $p\left(m_{1}, \ldots, m_{l}\right)$ where $p \in P_{2}$ and $m_{1}, \ldots, m_{l}$ are monomials. In other words, the $T$-ideal $I$ is $S$-generated by the finite set $P^{\prime}=P_{2}$.

Case 2. The ring $R$ is finitely generated. Let $r_{1}, \ldots, r_{s}$ be a finite system of generators for $R$. Since the elements $r_{1}, \ldots, r_{s}$ generate the ring $R$, each element $\alpha \in R$ can be written as a finite sum $\alpha=\sum \delta_{i} v_{i}$, where $\delta_{i}= \pm 1$ and the $v_{i}$ 's are (commutative) monomials in $r_{1}, \ldots, r_{s}$ (the monomials $v_{i}$ need not be distinct). Now we refine the foregoing linearization argument. We have shown that the $T$-ideal $I$ is generated as a two-sided ideal by the set of all polynomials of the form $p\left(\alpha_{1} m_{1}, \ldots, \alpha_{l} m_{l}\right)$, where $p \in P_{1}, \alpha_{1}, \ldots, \alpha_{l} \in R, l$ is an integer, and $m_{1}, \ldots, m_{l}$ are monomials. Write each of the elements $\alpha_{1}, \ldots, \alpha_{l}$ as a finite sum of monomials in $r_{1}, \ldots, r_{s}$ with coefficients \pm 1 . Since the set $P_{1}$ is closed under taking linearizations, applying the linearization argument once more we get that the $T$-ideal $I$ is generated as a two-sided ideal by the set of all polynomials of the form $p\left( \pm v_{1} m_{1}, \ldots, \pm v_{l} m_{l}\right)$, where $p \in P_{1}, l$ is an integer, $v_{1}, \ldots, v_{l}$ are monomials in $r_{1}, \ldots, r_{s}$, and $m_{1}, \ldots, m_{l}$ are monomials in $x_{1}, x_{2}, \ldots$ Let $P_{2}$ be the set of all polynomials of the form $p\left((-1)^{i_{1}} x_{1}, \ldots,(-1)^{i_{k}} x_{k}\right)$, where $p \in P_{1}$, and $i_{1}, \ldots, i_{k} \in\{0,1\}$. Since the set $P_{1}$ is finite, the set $P_{2}$ is also finite. So, the $T$-ideal $I$ is generated as a two-sided ideal by the set of all polynomials of the form $p\left(v_{1} m_{1}, \ldots, v_{l} m_{l}\right)$, where $p \in P_{2}, l$ is an integer, $v_{1}, \ldots, v_{l}$ are monomials in $r_{1}, \ldots, r_{s}$, and $m_{1}, \ldots, m_{l}$ are monomials in $x_{1}, x_{2}, \ldots$. Now let $P_{3}$ be the set of all homogenizations of polynomials from $P_{2}$ relative to $r_{1}, \ldots, r_{s}$ together with polynomials from $P_{2}$ themselves. The set $P_{3}$ is finite, since a polynomial has only finitely many homogenizations relative to a finite subset of $R$. Rewrite the formula (3) as

$$
\begin{aligned}
& p\left(x_{1}, \ldots, x_{i-1}, r_{j} x_{i}, x_{i+1}, \ldots, x_{l}\right) \\
& =r_{j}^{d_{i}} p\left(x_{1}, \ldots, x_{i-1}, x_{i}, x_{i+1}, \ldots, x_{l}\right) \\
& -q\left(x_{1}, \ldots, x_{i-1}, x_{i}, x_{i+1}, \ldots, x_{l}\right) .
\end{aligned}
$$

Note that if $p \in P_{3}$, then $q \in P_{3}$ and formula (6) shows that the polynomial $p\left(x_{1}, \ldots, x_{i-1}, r_{j} x_{i}, x_{i+1}, \ldots, x_{l}\right)$ belongs to the $S$-ideal generated by $P_{3}$. By induction on the total degree of the monomials $v_{1}, \ldots, v_{l}$ one shows that a polynomial of the form $p\left(v_{1} m_{1}, \ldots, v_{l} m_{l}\right)$, where $p \in P_{2}, v_{1}, \ldots, v_{l}$ are monomials in $r_{1}, \ldots, r_{s}$, and $m_{1}, \ldots, m_{l}$ are monomials in $x_{1}, x_{2}, \ldots$, can be written as an $R$-linear combination of polynomials of the form $p\left(m_{1}, \ldots, m_{j}\right)$, where $p \in P_{3}$, and $m_{1}, \ldots, m_{j}$ 
are monomials in $x_{1}, x_{2}, \ldots$ (To make the induction step, suppose $v_{j}=r_{j} \cdot v_{j}^{\prime}$ is a nontrivial factorization of $v_{j}$. Then set in (6) $x_{1}=v_{1} m_{1}, \ldots, x_{i}=v_{j}^{\prime} m_{i}, \ldots$, $x_{l}=v_{l} m_{l}$.) Thus, in this case the $T$-ideal $I$ is generated as a two-sided ideal by the set of all polynomials of the form $p\left(m_{1}, \ldots, m_{l}\right)$, where $p \in P_{3}, l$ is an integer, and $m_{1}, \ldots, m_{l}$ are monomials in $x_{1}, x_{2}, \ldots$ In other words, the $T$-ideal $I$ is $S$-generated by the finite set $P^{\prime}=P_{3}$.

Conversly, if a finite set $S$-generates a $T$-ideal, then this set also certainly generates this $T$-ideal as a $T$-ideal.

Lemma 1 is proved.

Proof of Theorem 1. Let $p=p\left(x_{1}, \ldots, x_{n}\right)$ be an identity of the representation $\psi$. Then

$$
m \cdot p\left(\psi\left(a_{1}\right), \ldots, \psi\left(a_{n}\right)\right)=0 \text { for all } a_{1}, \ldots, a_{n} \in A, m \in M
$$

if and only if

$$
p\left(\phi\left(a_{1}\right), \ldots, \phi\left(a_{n}\right)\right)=0 \text { for all } a_{1}, \ldots, a_{n} \in A
$$

if and only if

$$
\phi\left(p\left(a_{1}, \ldots, a_{n}\right)\right)=0 \text { for all } a_{1}, \ldots, a_{n} \in A
$$

if and only if

$$
p\left(a_{1}, \ldots, a_{n}\right) \in \operatorname{Ker} \phi \text { for all } a_{1}, \ldots, a_{n} \in A
$$

if and only if

$$
p=p\left(x_{1}, \ldots, x_{n}\right) \text { is an identity of } A / \operatorname{Ker} \phi .
$$

This means that the $S$-ideal of identities of $\phi$ coincides with the $T$-ideal of identities of $A / \operatorname{Ker} \varphi$. Now Lemma 1 finishes the proof of the theorem.

\section{ACKNOWLEDGMENTS}

The author thanks M. V. Sapir (University of Nebraska, Lincoln, USA) and M. V. Volkov (Ural State University, Ekaterinburg, Russia) for many useful discussions, and the referee, whose constructive remarks have been adopted in the present paper.

\section{REFERENCES}

[VSh] Vovsi S.M., Shon N.H. Identities of stable-by-finite representations of groups, Mat. Sbornik, 132(174), 1987, 578-591; English transl., Math. USSR Sb. 60 (1988), 569-581. MR 88f:20020

[AK] Al'shanskii L., Kushkuley A. Identities of the natural representation of the infinitely based semigroup, Proc. Amer. Math. Soc. 118, 1993, 931-938. MR 93i:20071

[S] Sapir M.V., Problems of Burnside type and the finite basis property in varieties of semigroups, Izv. AN SSSR, Ser.Mat., 51(2), 319-340, 1987; transl. in Math USSR.Izv, 30(2), 1988, 295-314. MR 88h:20078

[L] Lvov I.V., On varieties of associative rings I, Algebra and Logic, 12 (1973), 150-167. MR 52:10802

[K] Kruse R.L., Identities satisfied by a finite ring, J.Algebra, 26, 298-318. MR 48:4025 
[R1] Rowen L.H., Polynomial identities in ring theory, Academic Press, New York, 1980. MR 82a:16021

[R2] Rowen L.H., Ring theory, v.2, Academic Press, San Diego, 1988. MR 89h:16001

Department of Mathematics and Statistics, University of Nebraska-Lincoln, LinCOLN, NeBraska 68588-0323

E-mail address: nsilkin@math.unl.edu

Current address: 1326 Stevenson Center, Department of Mathematics, Vanderbilt University, Nashville, Tennessee 37240

E-mail address: nsilkin@math.vanderbilt.edu 